\title{
Oocyst wall formation and composition in coccidian parasites
}

\author{
Kelly Mai, Philippa A Sharman, Robert A Walker, Marilyn Katrib, David De Souza, \\ Malcolm J McConville, Michael G Wallach, Sabina I Belli, David JP Ferguson, Nicholas C Smith/ ${ }^{+}$
}

Institute for the Biotechnology of Infectious Diseases, University of Technology, PO Box 123 Sydney, Australia

The oocyst wall of coccidian parasites is a robust structure that is resistant to a variety of environmental and chemical insults. This resilience allows oocysts to survive for long periods, facilitating transmission from host to host. The wall is bilayered and is formed by the sequential release of the contents of two specialized organelles - wall forming body 1 and wall forming body 2 - found in the macrogametocyte stage of Coccidia. The oocyst wall is over $90 \%$ protein but few of these proteins have been studied. One group is cysteine-rich and may be presumed to crosslink via disulphide bridges, though this is yet to be investigated. Another group of wall proteins is rich in tyrosine. These proteins, which range in size from 8-31 kDa, are derived from larger precursors of 56 and $82 \mathrm{kDa}$ found in the wall forming bodies. Proteases may catalyze processing of the precursors into tyrosine-rich peptides, which are then oxidatively crosslinked in a reaction catalyzed by peroxidases. In support of this hypothesis, the oocyst wall has high levels of dityrosine bonds. These dityrosine crosslinked proteins may provide a structural matrix for assembly of the oocyst wall and contribute to its resilience.

Key words: Coccidia - oocyst - dityrosine crosslinking - Toxoplasma - Eimeria

Coccidian parasites, which include the genera Toxoplasma, Neospora, Hammondia, Isospora, Sarcocystis and Eimeria, amongst others, share many features. For example, like all Apicomplexa, their lifecycle includes asexual stages (sporozoites and merozoites) that possess a phylum-defining feature, the apical complex of organelles, associated with cell invasion. An additional defining feature of the Coccidia is the oocyst; coccidian parasites are transmitted from host to host by accidental ingestion of oocysts that contaminate food or water. Oocysts are remarkably hardy and able to persist in the environment for prolonged periods of time. The "softbodied" parasites are safely encapsulated inside a unique structure, the oocyst wall.

The oocyst wall is extremely robust. It is resistant to mechanical and chemical damage; breaking oocysts for laboratory studies requires prolonged, high-speed vortexing with glass beads and oocysts are routinely cleaned with bleach and stored in the harsh oxidant, potassium dichromate, or the mineral acid, sulphuric acid (Dubey et al. 1970, Shirley 1995, Schares et al. 2005). The wall is also resistant to proteolysis and impermeable to watersoluble substances, including many detergents and disinfectants (Monné \& Hőnig 1954, Ryley 1973). This contributes to the difficulty experienced in attempting to exclude oocysts from, for example, poultry houses.

Financial support: Australian Research Council's Discovery and Linkage Projects funding schemes (DP0664013, LP0454145), Abic Ltd (Israel), Rural Industries Research and Development Corporation's Postgraduate Scholarship Scheme, ARC/NHMRC research Network for parasitology (RN0460368)

+ Corresponding author: nick.smith@uts.edu.au

Received 16 October 2008

Accepted 4 December 2009
The oocyst wall is, however, permeable to some small molecules and lipid+soluble substances; ammonia and methyl bromide being two of the best known (Monné \& Hőnig 1954, Ryley 1973, Kuticic \& Wikerhauser 1996).

The oocyst wall is essentially consistent in structure across different species of coccidian parasites (Belli et al. 2006), but it is the oocyst wall of Eimeria that has been best studied, largely because of the relative ease of acquiring large numbers of oocysts of the parasites of this genera.

\section{Oocyst wall biogenesis}

The oocyst wall is formed from the contents of two specific organelles, wall forming bodies Type 1 (WFB1) and 2 (WFB2), found exclusively in the sexual, macrogamete stage of coccidian parasites (Scholtyseck et al. 1969, Scholtyseck 1973, Ferguson et al. 1975, 2003, Pittilo \& Ball 1980, Belli et al. 2006). In the early stages of macrogamete biogenesis, the macrogametocyte contains a centrally-located nucleus and numerous spherical deposits of electron dense material within swollen regions of the rough endoplasmic reticulum; these represent the early WFB2. Shortly after this, in the mid-stage of macrogamete development, spherical electron dense granules appear in the cytoplasm. These secretory-like granules arise from material that traffics from the Golgi bodies and represent the early WFB1 (Ferguson et al. 2003). Also present in the early/mid and mid-stage of development are an additional group of membrane bound vacuoles termed the veil forming bodies (Ferguson et al. 2000, 2003).

The veil forming bodies vary in appearance between species, being electron dense in Toxoplasma gondii (Ferguson et al. 2000) and electron lucent in Eimeria maxima (Ferguson et al. 2003). They are secreted during macrogamete maturation and are responsible for the formation of a veil on the outer surface of the maturing macrogamete and oocyst (Ferguson et al. 2000, 2003). 
The outer veil of E. maxima stains positively with antibodies that are specific for the apple domains of $T$. gondii microneme protein 4 , a protein associated with oocyst wall formation in this parasite. This suggests that the veil contains MIC4-like proteins and that the structure of the veil is conserved across the Coccidia (Ferguson et al. 2000, 2003). The veil is lost when oocysts are excreted from the host (Ferguson et al. 1975, Pittilo \& Ball 1980); therefore it is thought that the outer veil plays no role in protection of parasites during passage out of the host or in the external environment (Belli et al. 2006). Its precise role is still unknown, though it seems possible that it provides a temporary "frame" under which the oocyst wall is assembled. This idea is supported by the fact that the veil remains in place throughout the entire intracellular development phase of the oocyst (Pittilo \& Ball 1980).

WFB1 in mature macrogametocytes are large, spherical, membrane bound structures with electron dense contents (Pittilo \& Ball 1979, Ferguson et al. 2003, Belli et al. 2006). They are located in the periphery of the parasites and have long been thought to contain mucoproteins, mucopolysaccharides and glycoproteins (McLaren 1969, Scholtyseck 1973). The size of WFBs is species-specific. For example, WFB1 are larger than WFB2 in E. maxima, Eimeria tenella, Eimeria stieda and Eimeria perforans, whereas in Eimeria bovis, Eimeria falciformis and $T$. gondii, the reverse is true (Scholtyseck 1973). WFB2 are less electron dense than WFB1, have a less distinct structure (Ryley 1973, Scholtyseck 1973) and appear as spherical dilations enclosed within the rough endoplasmic reticulum (Ferguson et al. 1975, 2003, Pittilo \& Ball 1979). In the mature macrogametocyte, WFB1s locate in the periphery of the parasite inter-mixed with WFB2. A large number of polysaccharide granules, containing amylopectin and some lipid droplets are also seen in the cytoplasm of the mature macrogametocyte (Ferguson et al. 1975, Pittilo \& Ball 1980).

Once fertilized by a microgamete, a macrogamete develops into a zygote. The oocyst wall starts to form shortly afterwards (Pittilo \& Ball 1980), with WFB1s aligning beneath the limiting membrane of the zygote cytoplasm. A recent study (Ferguson et al. 2003) of oocyst wall formation in E. maxima, using immunocytochemistry with antibodies specific for WFBs, adds compelling evidence to support the mechanism of oocyst wall formation originally proposed by Pittilo and Ball in 1980. Thus, Ferguson et al. (2003) showed that oocyst wall formation is a sequential release of the contents of: (i) the veil forming bodies, (ii) WFB1 and (iii) WFB2. It is thought that this mechanism may be controlled at the level of the rough endoplasmic reticulum/ Golgi apparatus. Thus, in the mature macrogametocyte or zygote, after the veil has been laid down, WFB1 relocate to the periphery of the parasite. WFB1s quickly disaggregate and appear to fuse together at the surface of the parasite, ultimately forming the outer layer of the oocyst wall. Shortly after WFB1 form the outer layer, the WFB2s, located in the rough endoplasmic reticulum are transferred via the Golgi body and secretory granules to the surface where they fuse to create the inner layer of the oocyst wall.
The mature oocyst wall is bilayered. Initially, the outer layer may be as thick as $500-600 \mathrm{~nm}$ but, as the development of the wall progresses, the outer layer is compacted to $200 \mathrm{~nm}$ or less. An inner zone of approximately $40 \mathrm{~nm}$ separates the outer and inner layers, the latter layer also ultimately being $\sim 40 \mathrm{~nm}$ thick (Ferguson et al. 2003). The electron dense outer layer is variable in size across different genera; for example, it can be $\sim 200 \mathrm{~nm}$ thick in E. maxima (Ferguson et al. 2003) but is only 20-40 nm thick in T. gondii (Ferguson et al. 1975, 2000). The electron lucent inner layer, formed from the contents of WFB2, is more consistent, being around $40 \mathrm{~nm}$ thick in most genera studied (Belli et al. 2006). The two layers do not seem to be tightly fused and can be separated relatively easily (Monné \& Hőnig 1954); bleach treatment, for instance, usually strips the outer layer away from the inner layer.

\section{Biochemical composition of the oocyst wall}

The first serious microscopic and chemical examination of the oocyst wall (of E. maxima) was conducted by Monné and Hönig (1954), who used a number of destructive treatments that led them to conclude that the outer layer of the oocyst wall contained mainly quinone-tanned proteins without lipids, since the outer layer reacted with ammoniacal silver nitrate solution (an indication of quinones). They also noted that the outer layer was stripped off upon exposure to sodium hypochlorite, whereas the structure of the inner layer remained unchanged, leading them to conclude that the inner layer consisted of a lipid-protein matrix; they believed that lipids were bound firmly to proteins, thus protecting the inner layer against disintegration by sodium hypochlorite.

The first true biochemical examination of the oocyst wall was carried out by Ryley in 1973 using E. tenella. Ryley (1973) also noted that the outer layer was removed by sodium hypochlorite and found that it contained carbohydrates and proteins, with high proline content, whereas the inner layer consisted of $1.5 \%$ carbohydrates, $30 \%$ lipids and $70 \%$ proteins. The lipid in the inner layer was extractable in chloroform and appeared to be a mixture of "waxes" containing very small amounts of nitrogen and phosphorus. However, there are some limitations in this report: first, it did not show detailed analyses of the experimental work and, second, it did not document the metabolites detected in the inner wall.

The most recent biochemical analysis of the oocyst wall was conducted by Stotish et al., in 1978, using unsporulated oocysts isolated from the caeca of chickens infected with $E$. tenella. These researchers proposed that lipids were concentrated in the outer layer whereas the inner layer was composed of mainly glycoproteins. In addition, compositional analysis of the oocyst wall by gas-liquid chromatography and SDS-PAGE indicated that it was $19 \%$ carbohydrates, $14 \%$ lipids and $67 \%$ protein (Stotish et al. 1978). Lipids identified from the walls included a number of fatty alcohols, steroids and fatty acids such as docosanol, tetracosanol, hexacosanol, myristate, palmitate, stearate, oleate, linoleate and phospholipids. Protein content in the oocyst wall consisted of a repeating subunit of $\sim 10 \mathrm{kDa}$. Four carbohydrates 
- mannose, galactose, glucose and hexosamine - were detected. However, some doubts surround the validity of these conclusions because Stotish et al. (1978) noted that sodium hypochlorite treatment did not affect the structure of the oocyst walls from E. tenella unsporulated oocysts and this was a major basis for their conclusion that lipids are concentrated in the outer layer of the wall (since lipids would be expected to give some protection against stripping by sodium hypochlorite). In direct contradiction to the observations of Stotish et al. (1978), it has been shown that sodium hypochlorite treatment does have an effect on the structure of the oocyst wall in both E. maxima and E. tenella - the outer layer is stripped off (Monné \& Hőnig 1954, Nyberg et al. 1968, Nyberg \& Knapp 1970, Ryley 1973, Belli et al. 2006). Therefore, the biochemical compositions assigned to the two layers of the oocyst wall of E. tenella by Stotish et al. (1978) could be erroneous. We have, therefore, reinvestigated the biochemical composition of the oocyst wall using more reliable sensitive modern methods.

We isolated oocyst walls from intact E. maxima and E. tenella oocysts as published previously by Stotish et al. (1978). Oocyst walls (equivalent to the wall preparations isolated from $2 \times 10^{6}$ oocysts) were then resuspended in $100 \mu \mathrm{L}$ of distilled water and treated with alphaamylase to eliminate polysaccharide granules according to the protocol from Ryley (1973), with slight modification. Briefly, alpha-amylase was added to a final concentration of $0.5 \mathrm{mg} / \mathrm{mL}$ and $1 \mathrm{mg} / \mathrm{mL}$ for comparison and samples were incubated at $20^{\circ} \mathrm{C}$ overnight. On the next day, the samples were centrifuged at $10,000 \mathrm{~g}$ for 10 min at $4^{\circ} \mathrm{C}$, the supernatant was discarded and the pellet was washed in distilled water. The washing step was repeated three times. The effect of alpha-amylase on elimination of polysaccharide granules was investigated by microscopic examination and transmission electron microscopy (TEM) as published previously by Ferguson et al. (2003), with a minor modification. Briefly, each sample of oocyst wall fragments was centrifuged and the pellets were fixed in $4 \%$ glutaraldehyde in $0.1 \mathrm{M}$ phosphate buffer. The pellets were post-fixed in $1 \%$ osmium tetroxide in phosphate buffer, dehydrated in absolute ethanol, treated with propylene oxide and embedded in Spurr's epoxy resin. Thin sections of oocyst walls were then mounted on copper grids and stained with uranyl acetate and lead citrate for routine electron microscopy. In addition, some sections were mounted on formvarcoated gold grids and stained with silver methenamine. The sections were floated on drops of a freshly prepared and filtered mixture containing silver nitrate and hexamethylene tetramine in a borax-based buffer ( $\mathrm{pH} 9.2$ ). Sections were stained in the dark at $40^{\circ} \mathrm{C}$ for $60 \mathrm{~min}$ and washed in water prior to examination. In both cases, sections were examined in a Jeol 1200EX TEM.

In the absence of treatment with alpha-amylase, the purified oocyst walls appeared to be heavily contaminated with polysaccharide granules (Fig. 1A, B). When alpha-amylase was added to the oocyst walls, the number of polysaccharide granules was reduced markedly, as shown in the sample treated with $0.5 \mathrm{mg} / \mathrm{mL}$ alpha-amylase (Fig. 1C) and, more especially, in the sample treated with $1 \mathrm{mg} / \mathrm{mL}$ alpha-amylase (Fig. 1D). Examination by light and UV microscopy (Fig. 1E, F, respectively) confirmed the characteristic blue autofluorescence of the purified oocyst walls after alpha-amylase treatment, indicating that the basic chemical structure of the walls was not altered significantly by the treatment.

The oocyst walls prepared from E. tenella and $E$. maxima sporulated and unsporulated oocysts were examined by TEM to confirm the presence of the inner and/or outer layers. The outer layer of the oocyst walls (both E. tenella and E. maxima), appeared electrondense with a roughened appearance on the outer surface, and the inner layer was electron-lucent (Fig. 2A-C, E, G), observations that are consistent with those of Ferguson et al. (2003). The oocyst walls from unbleached samples remained intact (retaining a bi-layered structure) whereas in bleached samples, generally only the inner layer was seen (Fig. 2D, F, H), confirming the observations that bleaching does strip the outer layer of oocyst walls (Monné \& Hőnig 1954, Nyberg et al. 1968, Nyberg \& Knapp 1970, Ryley 1973, Belli et al. 2006). There was a single exception to this pattern; walls prepared from a sample of bleached E. tenella sporulated oocysts remained intact as a bi-layered structure (Fig. 2B). This was not always observed and the reason for this exception is not known. However, this exceptional sample proved very useful in subsequent comparisons of wall composition.

The compositional analysis of bleached oocyst walls was carried out as published previously by McConville et al. (1990), with a minor modification (unbleached samples were not analysed due to the presence of various contaminants, including residual amylopectin, which would distort the analyses). Briefly, the oocyst walls

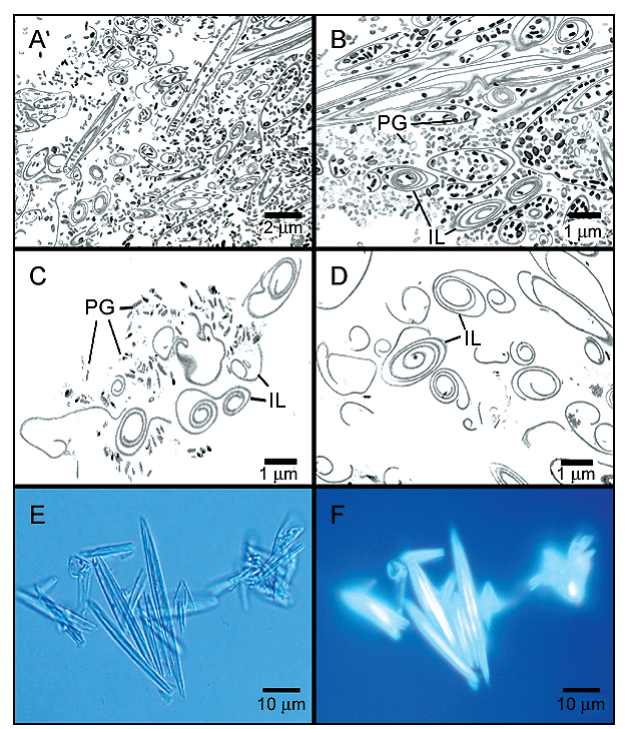

Fig. 1: micrographs of alpha-amylase treated oocyst walls prepared from Eimeria maxima bleached unsporulated oocysts. Each sample contained oocyst walls equivalent to $2 \times 10^{6}$ E. maxima unsporulated oocysts in the absence of alpha-amylase (A, B) or treated with alphaamylase to a final concentration of $0.5 \mathrm{mg} / \mathrm{mL}$ (C) or $1 \mathrm{mg} / \mathrm{mL}$ (D). E: purified, alpha-amylase treated oocyst walls under bright field; F: purified, alpha-amylase treated oocyst walls under UV light; IL: inner layer of oocyst walls; PG: polysaccharide granules. 


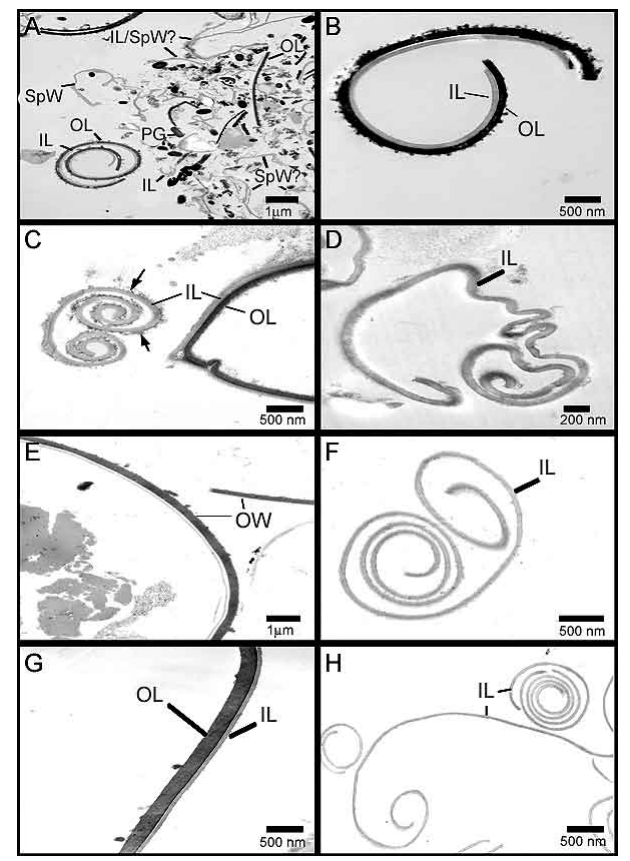

Fig. 2: electron micrographs showing the effect of bleach on oocyst wall (OW) preparations from Eimeria tenella and Eimeria maxima. A, B: E. tenella unbleached and bleached sporulated OW, respectively; C, D: E. tenella unbleached and bleached unsporulated OW, respectively [arrows in $\mathrm{C}$ indicate hydrolyzed polysaccharide granules (PG) attached to the OW]; E, F: E. maxima unbleached and bleached sporulated OW, respectively; G, H: E. maxima unbleached and bleached unsporulated OW, respectively; IL: inner layer of OW; OL: outer layer of OW; SpW: sporocyst walls.

were resuspended in $50 \mu \mathrm{L}$ of 1:1 chloroform:methanol and transferred into a clean glass capillary tube with one end flame sealed. Heptadecanoic acid (C17:0; $10 \mathrm{~nm})$ was added to the capillary tube as an internal standard for subsequent quantification of metabolites. The sample was then dried in vacuo at $50^{\circ} \mathrm{C}$. This step was repeated again in the presence of methanol to enable the complete removal of $\mathrm{H}_{2} \mathrm{O}$. Methanolic $\mathrm{HCl}(0.5 \mathrm{M} \mathrm{HCl}$ in methanol; $50 \mu \mathrm{L}$ ) was added to each capillary tube and sealed under vacuum. The sample was then incubated at $80^{\circ} \mathrm{C}$ overnight. Following methanolysis, the sample was cut open and the acid was neutralized by the addition of $10 \mu \mathrm{L}$ pyridine. The neutralized solution was then transferred to a GC-MS vial insert and dried in vacuo. Trimethylsilyl (N-methyl-N-trifluroacetamide + $1 \%$ trimethylchlorosilane; $50 \mu \mathrm{L}$ ) reagent was added to the tube, which was gently flicked to ensure the sample was well mixed. The sample was then transferred into an insert vial of autosampler vials for compositional analyses of carbohydrates and lipids by gas chromatography (GC; Agilent 6890N GC) and mass spectrometry (MS, Agilent 5973 Mass Selective Detector).

Oocyst wall samples were also hydrolyzed in $6 \mathrm{~N} \mathrm{HCl}$ as published previously (McConville et al. 1990), with a minor modification. Briefly, the oocyst walls were resuspended in $100 \mu \mathrm{L}$ of 1:1 chloroform:methanol and transferred into GC-MS vial inserts containing $1 \mathrm{~nm}$ of scyllo-inositol as an internal control for subsequent quantification of metabolites. The sample was dried in vacuo at $55^{\circ}$. The vial inserts were then transferred into reaction vials containing $200 \mu \mathrm{L} 6 \mathrm{~N} \mathrm{HCl}$, followed by evacuation under nitrogen. The samples were capped immediately to prevent oxidation by air and incubated at $110^{\circ} \mathrm{C}$ over night. The vial inserts were then placed into $\mathrm{GC}-\mathrm{MS}$ vials and the residual $\mathrm{HCl}$ was evaporated under nitrogen. Vial contents were then incubated for $2 \mathrm{~h}$ at $\mathrm{rt}$ in $20 \mu \mathrm{L}$ pyridine to allow resuspension and neutralization of $\mathrm{HCl}$ contaminants. N,O-bis[trimethylsilyl] trifluoroacetamide $+1 \%$ trimethylchlorosilane $(20 \mu \mathrm{L})$ was added to enable trimethylsilyl derivatisation and the reactions were carried out at $80^{\circ} \mathrm{C}$ for $30 \mathrm{~min}$. The amino acid composition of the oocyst wall proteins was analyzed by GC-MS.

Finally, oocyst wall proteins were extracted using the method described by Meyers et al. (1998). Oocyst walls (from $2 \times 10^{6}$ oocysts) were resuspended in $100 \mu \mathrm{L}$ of 1 $\mathrm{M} \mathrm{NaOH}$, followed by boiling for $10 \mathrm{~min}$ to solubilize the wall proteins. The lysate was then cooled down at rt for $10 \mathrm{~min}$. Twenty microliters of $5 \mathrm{M} \mathrm{HCl}$ was added to neutralize the lysate, which was then brought up to $1 \mathrm{~mL}$ by the addition of $880 \mu$ LPBS. Absorbance at $230 \mathrm{~nm}$ and $260 \mathrm{~nm}$ was measured using a spectrophotometer (GeneQuant, Amersham Biosciences) and protein concentration was calculated using the following equation: $\left\{[\right.$ Protein $\left.]=\left(183 \times \mathrm{A}_{230}\right)-\left(75.8 \times \mathrm{A}_{280}\right)\right\}$.

Our analyses revealed remarkable similarities between all the oocyst wall samples (Table I). Thus, the relative levels, on a percentage $\mathrm{w} / \mathrm{w}$ basis of carbohydrate, lipid and protein were contained in relatively narrow ranges of $0.6-2.0 \%$ carbohydrate, $1.4-7.6 \%$ lipid and $90.4-98.3 \%$ protein, for unsporulated or sporulated oocysts, from E. tenella or E. maxima. It is perhaps noteworthy, however, that the lower values for carbohydrates and lipids were seen in the sporulated oocyst samples from both E. tenella and E. maxima. This indicates that the single bilayered oocyst wall sample (the sporulated oocysts of $E$. tenella) did not exhibit a distinctly different composition than the single layered sporulated oocyst wall sample from E. maxima. In contrast to these results, the percentages of carbohydrate, lipid and protein reported by Stotish et al. (1978) for unsporulated oocysts of $E$. tenella are quite different, with only $67 \%$ protein and $19 \%$ carbohydrate and $14 \%$ lipid being recorded.

Analysis of $6 \mathrm{~N} \mathrm{HCl}$ hydrolysates of the oocyst walls of E. tenella and E. maxima revealed that essentially all twenty amino acids are present in the walls of sporulated and unsporulated oocysts. A limited qualitative comparison of five of the more abundant amino acids, isoleucine (Ile), aspartic acid (Asp), valine (Val), proline (Pro) and arginine (Arg) was possible by assigning Ile a standardized value of 1.0 and presenting the other four amino acids as a ratio of this. It is important to not over interpret this data; it provides a limited snapshot of the relative order of abundance of these five amino acids but the ratio values probably do not very precisely reflect their abundance. When examined in this way, the relative abundance of the amino acids is similar in all the samples, whether E. tenella or E. maxima, unsporulated or sporulated oocysts, and is in the order Ala $>$ Pro $>$ Val $>$ Asp $>$ Ile. The only slight exception to this is the sporulated oocyst 
sample of E. maxima, where Asp and Val are reversed, but marginally. The results of Stotish et al. (1978) are in accord with these observations.

Sporulated oocysts of both species contained a number of fatty acids (saponifiable lipids) including palmitic acid (C16:0), stearic acid (C18:0), oleic acid (C18:1), linoleic acid (C18:2), behenic acid (C22:0), lignoceric acid (C24:0) (Table II). Non-saponifiable lipids such as cholestadiene, cholestane and cholesterol were also detected. Most of these were present in a similar w/w percentage in the two species except that the bilayered E. tenella sample was comprised of $44.7 \%$ oleic acid whereas the E. maxima sample was only $19.5 \%$ oleic acid. The E. tenella sample also had higher quantities of palmitic acid $(13.2 \%$ versus $5.9 \%$ in E. maxima). In apparent compensation of these differences, walls from $E$. tenella sporulated oocysts had lower relative percentages of linoleic acid $(2.1 \%$ in $E$. tenella versus $8.2 \%$ in E. maxima), lignoceric acid (1.8\% versus $5.4 \%)$ and, especially, cholestane $(3.5 \%$ versus $11 \%)$ and cholesterol (12.1\% versus $24.2 \%)$ than the $E$. maxima sporulated oocyst walls.

The levels of lipids in unsporulated oocysts of $E$. tenella and E. maxima were quite similar to each other but differed from the sporulated wall samples in that they totally lacked linoleic acid. The relative levels of oleic acid in the unsporulated oocyst samples resembled that seen in the E. maxima sporulated oocyst sample and, so, were also different from the levels apparent in the bilayered E. tenella sporulated oocyst sample. The data from Stotish et al. (1978) for lipid composition of unsporulated oocyst walls of $E$. tenella did not resemble the observations made here using GC-MS. First, the samples analysed by Stotish et al. (1978) contained no behenic acid, lignoceric acid, cholestadiene or cholestane. Second, Stotish et al. (1978) reported the presence of myristate, as well as several fatty alcohols (docosanol, tetracosanol, hexacosanol, octacosanol and triacosanol)

\section{TABLE I}

The biochemical composition of the oocyst wall of Eimeria

\begin{tabular}{|c|c|c|c|}
\hline \multicolumn{4}{|c|}{$\begin{array}{l}\text { Eimeria tenella oocyst walls } \\
\quad \% \text { of metabolites }(\mathrm{w} / \mathrm{w})\end{array}$} \\
\hline & \multicolumn{3}{|c|}{ Metabolite $^{a}$} \\
\hline Sample & Carbohydrate & Lipid & Protein \\
\hline Walls from $\mathrm{S} / \mathrm{O}$ & 0.6 & 1.6 & 97.8 \\
\hline Walls from $\mathrm{U} / \mathrm{O}$ & 1 & 4.4 & 94.6 \\
\hline \multicolumn{4}{|c|}{$\begin{array}{l}\text { Eimeria maxima oocyst walls } \\
\quad \% \text { of metabolites }(\mathrm{w} / \mathrm{w})\end{array}$} \\
\hline & \multicolumn{3}{|c|}{ Metabolite $^{a}$} \\
\hline Sample & Carbohydrate & Lipid & Protein \\
\hline Walls from $\mathrm{S} / \mathrm{O}$ & 0.3 & 1.4 & 98.3 \\
\hline Walls from $\mathrm{U} / \mathrm{O}$ & 2.0 & 7.6 & 90.4 \\
\hline
\end{tabular}

not detected here; Stotish et al. (1978) reported hexacosanol and phospholipids as being especially abundant.

The carbohydrate content of E. tenella sporulated oocyst walls was very similar to that of sporulated oocyst walls of E. maxima, the carbohydrate content being made up of $4.3-5 \%$ mannose, $33.7-37.4 \%$ galactose and $58.3-61.3 \%$ glucose (the latter possibly reflecting residual contamination with amylopectin). Likewise, the carbohydrate profile of unsporulated oocyst walls of E. tenella was similar to that of unsporulated oocyst walls of $E$. maxima but quite different to that of the sporulated walls, being dominated by galactose ( $62.3 \%-67.6 \%)$, followed by glucose (26-27.9\%) and mannose (6.4-9.8\%). The carbohydrate composition of unsporulated oocysts of E. tenella reported by Stotish et al. (1978) was, however, very different; they reported that the carbohydrate profile of the wall was $79.6 \%$ glucose and $11 \%$ hexosamine (which was not detected in the GC-MS analysis reported here) and only $7.2 \%$ galactose and $2.2 \%$ mannose.

Thus, to summarise, GC-MS analyses of oocyst walls of E. tenella and E. maxima indicates that they are primarily made up of protein (greater than $90 \%$ ) with relatively small amounts of lipid and carbohydrate. The composition of the oocyst walls of both species was very

TABLE II

The lipid composition of the oocyst wall of Eimeria

\begin{tabular}{|c|c|c|}
\hline \multicolumn{3}{|c|}{$\begin{array}{l}\text { Eimeria tenella oocyst walls } \\
\% \text { of metabolites }{ }^{a}(\mathrm{w} / \mathrm{w})\end{array}$} \\
\hline \multirow[b]{2}{*}{ Lipid } & \multicolumn{2}{|c|}{ Sample } \\
\hline & Walls from $\mathrm{S} / \mathrm{O}$ & Walls from $\mathrm{U} / \mathrm{O}$ \\
\hline Palmitic acid (C16:0) & 13.2 & 13.3 \\
\hline Stearic acid (C18:0) & 13.7 & 22.2 \\
\hline Oleic acid (C18:1) & 44.7 & 16.1 \\
\hline Linoleic acid (C18:2) & 2.1 & 0 \\
\hline Behenic acid (C22:0) & 4.5 & 9.8 \\
\hline Lignoceric acid (C24:0) & 1.8 & 2.9 \\
\hline Cholestadiene & 4.4 & 7.2 \\
\hline Cholestane & 3.5 & 16.7 \\
\hline Cholesterol & 12.1 & 11.8 \\
\hline \multicolumn{3}{|c|}{$\begin{array}{l}\text { Eimeria maxima oocyst walls } \\
\% \text { of metabolites }{ }^{a}(\mathrm{w} / \mathrm{w})\end{array}$} \\
\hline & \multicolumn{2}{|c|}{ Sample } \\
\hline Lipid & Walls from $\mathrm{S} / \mathrm{O}$ & Walls from $\mathrm{U} / \mathrm{O}$ \\
\hline Palmitic acid (C16:0) & 5.9 & 11.7 \\
\hline Stearic acid (C18:0) & 14.4 & 21.4 \\
\hline Oleic cid (C18:1) & 19.5 & 18.4 \\
\hline Linoleic acid (C18:2) & 8.2 & 0 \\
\hline Behenic acid (C22:0) & 6.4 & 9.6 \\
\hline Lignoceric acid (C24:0) & 5.4 & 10.9 \\
\hline Cholestadiene & 5.0 & 6.4 \\
\hline Cholestane & 11.0 & 7.0 \\
\hline Cholesterol & 24.2 & 14.6 \\
\hline
\end{tabular}

$a$ : relative levels of lipids prepared from $10^{6}$ sporulated $(\mathrm{S} / \mathrm{O})$ or unsporulated oocysts (U/O). 
similar, whether sporulated or unsporulated, though there was perhaps slightly more protein (and, resultingly, proportionally less carbohydrate and lipid) in the sporulated oocyst walls. This may reflect the presence of sporocyst walls, which were occasionally seen in the sporulated oocyst wall samples (Fig. 2A) and, if so, indicates that the composition of the sporocyst wall is slightly different to that of the oocyst wall. Surprisingly, the presence of both the outer and inner layer of the oocyst wall (as in the E. tenella sporulated oocyst wall sample) did not greatly affect the overall composition of the wall, indicating that both layers are chemically similar, even though their relative electron densities are different, as indicated by the TEM images.

All of the above results are at odds with those of Stotish et al. (1978), who reported much lower protein content and much higher quantities of carbohydrate and lipid in the unsporulated oocyst wall of $E$. tenella. These discrepancies can almost certainly be ascribed to contamination of the walls with the internal contents of the oocyst, as the TEM images presented here document the presence of large numbers of amylopectin granules in the samples prepared following the method described by Stotish et al. (1978). The high levels of glucose reported by Stotish et al. (1978) - much higher than detected by the GC-MS analyses reported here - support this contention as amylopectin is a polymer of glucose monomers joined by alpha- 1,4 linkages (Ryley et al. 1969, 1973).

The lipid compositions of the oocyst wall samples analysed in this study are also different to those reported by Stotish et al. (1978), who failed to detect several of the lipids detected by the sensitive GC-MS methodology used here. Ironically, Stotish et al. also detected several fatty alcohols and phospholipids, as well as hexosamine, none of which were detected in this study. This could reflect the higher stringency of the analyses conducted in our study - GC-MS rules out false positives very effectively - but also probably betrays the fact that the wall samples prepared by Stotish et al. (1978) were contaminated with internal contents of the oocysts.

\section{Proteins of the oocyst wall}

The observation that the oocyst wall is dominated by protein is an important one as it implies that understanding the structure and characteristics of oocyst wall proteins - the major building blocks of the oocyst wall - will provide an insight into how the oocyst wall is formed and, potentially, identify vulnerabilities for attack by chemical or immunological agents either within the host and prior to oocyst wall formation or in the environment (e.g., floor litter of poultry houses).

There are only a small number of oocyst wall proteins that have been identified and characterized, mostly from Eimeria species. Stotish et al. (1978) believed that the protein content of the oocyst wall was predominantly, if not solely, a repeating subunit of $\sim 10 \mathrm{kDa}$, based on their detection of only a single band on SDS-PAGE gels. Karim et al. (1996) made similar conclusions when describing a monoclonal antibody to a single $12 \mathrm{kDa}$ protein band of the oocyst wall of $E$. tenella; the antibody reacted with macrogametocytes, as well as the inner wall of oocysts, and crossreacted with E. maxima. Eschenbacher et al. (1996) discovered $14 \mathrm{kDa}$ oocyst wall proteins in E. tenella and Eimeria acervulina, noting that these proteins were characterized by an abundance of amino acids that contain hydroxyl groups in their side-chains, especially serine, tyrosine and threonine. Based on this, they resurrected the idea, first proposed by Monné and Hőnig (1954), and reitereated by Pittilo and Ball (1980), that the oocyst wall contains quinone-tanned protein.

Studies on the macrogametocyte stage of Eimeria have yielded valuable information about oocyst wall proteins. Mouafo et al. (2002) reported that a monoclonal antibody raised against macrogametocytes of E. tenella, reacted with three proteins of 23,25 and $30 \mathrm{kDa}$ in the inner layer of the oocyst wall, in addition to an antigen of $51 \mathrm{kDa}$ in the macrogametocytes. In parallel, detailed studies on the 56 and $82 \mathrm{kDa}$ proteins from the macrogametocytes of E. maxima (EmGam56 and EmGam82) demonstrated (by N-terminal amino acid sequencing and Western blotting) that a series of oocyst wall proteins of 8, 10, 12 and $31 \mathrm{kDa}$ were all derived from these two precursor proteins (Belli et al. 2003). Like the 14 $\mathrm{kDa}$ proteins described by Eschenbacher et al. (1996), these proteins are tyrosine-rich (it seems likely that the 10,12 and $14 \mathrm{kDa}$ proteins described by Stotish et al. 1978, Eschenbacher et al. 1996, Mouafa et al. 2002, are essentially the same as the 8,10 and $12 \mathrm{kDa}$ proteins uncovered by Belli et al. 2003 using methods that enabled separation of an apparent single band on an SDS-PAGE gel into three bands). An additional protein of $\sim 29 \mathrm{kDa}$ was also noted but its composition was not defined.

Antibodies to EmGam56 and EmGam82 and to affinity purified gametocyte antigens (APGA) were subsequently used to definitively map the relocation of proteins from WFB1 to the outer layer of the oocyst wall and proteins from WFB2 to the inner layer of the oocyst wall of E. maxima (Ferguson et al. 2003). We have recently obtained similar results with both E. tenella and E. acervulina, demonstrating the conservation of these proteins and the process of wall formation (SI Belli et al., unpublished observations). Recently, Krücken et al. (2008) have confirmed the presence of two E. tenella homologues of EmGam56 and, additionally, reported the discovery of a $22 \mathrm{kDa}$ antigen in the macrogametocytes of E. tenella. Like the other proteins so far characterized, antibodies to this protein react with WFB2 and the inner layer of the oocyst wall. Although this $22 \mathrm{kDa}$ protein does contain some tyrosine residues, it is dominated by histidine and proline residues. It is notable for another reason, namely that its gene is present in extremely high copy number (in contrast to the single copies of the genes for, for example, EmGam56 and EmGam82), indicating that it may be important in oocyst wall formation via a mechanism distinct from that of the tyrosine-rich proteins. As yet, no information is available about whether this $22 \mathrm{kDa}$ protein is processed into smaller polypeptides nor how it is incorporated into the oocyst wall, though its involvement in stabilizing the oocyst wall via cross-links between histidine and catechols, as seen in insect cuticles (Christensen et al. 1991, Xu et al. 1997, Kerwin et al. 1999), is a distinct possibility (Krücken et al. 2008). 
The only other oocyst wall proteins to be documented thus far are the members of the multigene Cryptosporidium oocyst wall protein (COWP), a family of large (174-190 kDa), cysteine-rich proteins that localise to WFB1 of macrogametocytes and the inner wall of the Cryptosporidium oocyst (Spano et al. 1997, Templeton et al. 2004). Furthermore, the protein also localizes to a single large vesicle within immature macrogametocytes, indicating that it is a potential early indicator of sexual stage development.

It is thought that COWP forms extensive disulphide bridges and matrices within the oocyst wall (Spano et al. 1997). This would make it quite distinct from the other oocyst wall proteins described for Eimeria but this fits with the fact that the Cryptosporidium oocyst wall does not autofluoresce blue under UV light, in contrast to those of Eimeria, Toxoplasma, Neospora, Sarcocystis, Isospora and Cyclospora, which all display the blue autofluorescence characteristic of dityrosine bonds (Belli et al. 2006).

An exhaustive analysis of the Cryptosporidium parvum genome sequence revealed eight putative paralogues of COWP1, all displaying varying degrees of conservation of the Type I and Type II repeats (Templeton et al. 2004). While all nine members of this gene family appeared to contain multiple copies of the cysteine-rich, Type I repeats, only COWP1, 2 and 3 were observed to contain the Type II repeats. Furthermore, characteristic histidine-rich domains were observed in all $C$. parvum COWP sequences with the exception of COWP4 and COWP9. Semi-quantitative RT-PCR analyses revealed up-regulation in the transcription of all nine genes in parasite stages associated with sexual stage development and oocyst formation in C. parvum, supporting the hypothesis that these COWP proteins are involved in the formation of the oocyst wall.

The study by Templeton et al. (2004) also uncovered a homologue of COWP6 in the T. gondii genome sequence (referred to as $\operatorname{TgOWP1}$ ), potentially extending this wall-forming hypothesis to other coccidians. This hypothesis was also validated to some degree by the observation that no homologue to any of the COWP proteins could be found in the non-coccidian, apicomplexan, Plasmodium falciparum. Very recently, we have found two apparent homologues in Eimeria (Walker et al., unpublished observations). The role of these proteins in oocyst wall formation in Toxoplasma and Eimeria has yet to be investigated.

\section{Proteases, peroxidases, dityrosine bonds and oocyst wall formation}

A model for how the tyrosine-rich wall proteins are incorporated into the oocyst wall of Eimeria has been proposed by Belli et al. (2006), based on two well characterised gametocyte proteins, EmGam56 and EmGam82. Briefly, the model proposes that EmGam56 and EmGam82 are precursors that are processed into small tyrosine-rich wall proteins, perhaps by macrogametespecific proteases. The model goes on to propose that the tyrosine-rich proteins are oxidized, by peroxidase(s), and crosslinked via their tyrosine residues to form a matrix that subsequently becomes dehydrated ("tanned") and leads to hardening of the oocyst wall, with its accompanying, notorious resilience. This model generates several testable hypotheses: first, precursor proteins found in WFBs will be processed by gametocyte-specific proteases into smaller, tyrosine-rich peptides that are found in the oocyst wall, second, peroxidases will be specifically located in WFBs and will catalyse cross-linking of proteins via their tyrosine residues and, third, dityrosine bonds will be present in the oocyst wall. There is some evidence that all these hypotheses are correct.

As mentioned above, it has been shown that EmGam56 and EmGam82 are processed into small tyrosine-rich wall proteins of $8,10,12$ and $31 \mathrm{kDa}$ (Belli et al. 2003). Thus, antibodies to EmGam56 and EmGam82 also react with these smaller oocyst wall proteins and $\mathrm{N}$-terminal sequencing maps the location of these proteins within EmGam56 and EmGam82. It is easy to envisage that this processing is the result of proteolysis by an, as yet, unidentified protease or proteases. If proven, and the proteases that are responsible for the processing of these two gametocyte proteins are identified, then a totally new class of anticoccidial drug - a specific protease inhibitor (or inhibitors) - is not hard to imagine. Thus, future work directed at identifying proteases from the sexual stages of Eimeria is well warranted. Indeed, it has already commenced and our recent work has detected several gametocyte-specific proteases (Katrib et al., unpublished observations). Included amongst these are several subtilisins, which are particularly interesting with regard dityrosine bond formation because of their known role in the formation of the cuticle of nematodes. Thus, the assembly of cuticlins and collagens to form the cuticle involves a number of catalytic pathways: (i) collagens are synthesised as proproteins that are cleaved at the N-terminus by a subtilisin-like protease prior to cuticle formation (Thacker et al. 1995, 2000, 2006, Thacker $\&$ Rose 2000b), (ii) the collagens (and cuticlins) are held together by di- and tri-tyrosine crosslinks (Page \& Winter 2003) and (iii) dual oxidase is the oxidative enzyme responsible for the generation of the tyrosine crosslinks (Page \& Winter 2003). Mutations at any one of these steps, results in a deficiency of di and tri-tyrosine in the cuticle, the formation of a structurally defective cuticle and parasite death (Page \& Winter 2003). Our model of oocyst wall formation predicts a very similar picture.

It has also been shown that the macrogametocytes of E. maxima have high levels of activity of peroxidases and that this activity is exquisitely focused within the WFBs and developing oocyst wall of the parasite (Belli et al. 2003, 2006). An endogenous peroxidase has not yet been isolated from the WFBs of coccidian parasites but we have been able to demonstrate that exogenous peroxidases from horseradish or Arthromyces can induce crosslinking of EmGam56 in vitro, with accompanying formation of dityrosine bonds, as detected by HPLC with UV and visible detectors (Mai et al., unpublished observations).

Dityrosine bonds are readily demonstrable in the oocyst wall. Thus, oocysts of all coccidians are well known to autofluoresce blue under UV light in the excitation wavelength range of $330-385 \mathrm{~nm}$, a characteristic 
of dityrosine bonds (Belli et al. 2006). Furthermore, the oocyst wall of E. maxima contains remarkable levels of dityrosine and, to a lesser extent, 3,4-dihydroxyphenylalanine (DOPA), another derivative of tyrosine (Belli et al. 2003). These levels are much higher than many normal physiological situations and imply that the generation of dityrosine bonds in the oocyst wall is the result of a deliberate enzymatic process engineered by the parasite (Belli et al. 2006).

The hypothesis that dityrosine crosslinking of proteins is a key feature of oocyst wall formation and structure helps to explain the incredible environmental resilience of coccidian oocysts - quinone tanning and dityrosine crosslinking, leading to sclerotization, are widespread in the animal kingdom, and beyond, in association with the construction of structural matrices. Examples include such structures as insect cuticular resilin (Andersen 1964), exoskeletons of the nematodes, Haemonchus contortus, Caenorhabditis elegans and Ascaris suum (Fujimoto 1975, Fetterer \& Rhoads 1990, Lassandro et al. 1994), ascospore walls from the yeast, Saccharomyces cerevisiae (Briza et al. 1986), cell walls of Candida albicans (Smail et al. 1995), and the fertilization membrane of sea urchin eggs, Stronglycocentrotus purpuratus (Foerder \& Shapiro 1977). The presence of dityrosine in vertebrate animal proteins is also seen in chicken aorta elastin, collagen and connective tissues from bovines, rats and cats, and in tissues of normal and diseased humans (LaBella et al. 1968, Amado et al. 1984, Davies et al. 1999). DOPA-containing proteins are also widely distributed in nature and are involved in the synthesis of extraorganismic structural materials such as helminth worm and mosquito eggshells, egg capsules, cocoons, mussel byssal threads and various biological glues and cements (Waite 1990, Huggins \& Waite 1993). In addition, phenol oxidase activity (catalyzing oxidation of DOPA to dopachrome) is detected only in homogenates of female Trichuris suis (a parasite inhabiting the intestines of pigs), not males, suggesting that the enzyme is likely to be associated with hardening of eggshell and that the eggshell proteins might consist of DOPA-containing proteins (Fetterer \& Hill 1993). Understanding this process in oocysts may well lead to novel strategies to limit the transmission of coccidian parasites. In fact, there is probably already an outstanding example of this, in the form of a subunit vaccine against coccidiosis in chickens, CoxAbic ${ }^{\circledR}$.

CoxAbic ${ }^{\circledR}$ is the only commercially available subunit vaccine against a protozoan parasite. It has been used around the world in trials involving 177 million broiler chickens and the results from all trials clearly demonstrate that, in all aspects of parasite control, broiler growth, flock health and mortality, the performance of CoxAbic ${ }^{\circledR}$ broilers was similar to that of medicated or live vaccine control groups. Indeed, in most instances, CoxAbic ${ }^{\circledR}$ broilers performed slightly better than flocks reared on prophylactic drugs or live vaccines (M Wallach et al., unpublished observations). Cox $\mathrm{Abic}^{\circledR}$ is composed of an APGA preparation from E. maxima, dominated by EmGam56 and EmGam82 (Wallach 2002). Immunization of broiler breeder hens with the vaccine stimulates the production of IgG (sometimes referred to as $\operatorname{IgY}$ ) antibodies that are transferred to the developing broiler chick via the egg yolk. The results presented in this paper, together with previous studies on oocyst wall formation (Belli et al. 2003, Ferguson et al. 2003), are consistent with the hypothesis that the protective antibodies prevent formation of oocysts by either: (i) protecting the full length versions of EmGam56 and EmGam82 from degradation/proteolysis into smaller tyrosine-rich polypeptides destined for incorporation into the oocyst wall or (ii) interfering with the formation of dityrosine crosslinks between the tyrosine-rich polypeptides. Whether or not, this knowledge can be applied to control of other parasites remains to be seen.

\section{REFERENCES}

Amado R, Aeschbach R, Neukom H 1984. Dityrosine: in vitro production and characterization. Methods Enzymol 107: 377-388.

Andersen SO 1964. The crosslinks in resilin identified as dityrosine and trityrosine. Biochim Biophys Acta 93: 213-215.

Belli SI, Smith NC, Ferguson DJP 2006. The coccidian oocyst: a tough nut to crack! Trends Parasitol 22: 416-423.

Belli SI, Wallach MG, Luxford C, Davies MJ, Smith NC 2003. Role of tyrosine-rich precursor glycoproteins and dityrosine and 3,4-dihydroxyphenylalanine-mediated protein cross-linking in development of the oocyst wall in the coccidian parasite Eimeria maxima. Eukaryot Cell 2: 456-464.

Briza P, Winkler G, Kalchhauser H, Breitenbach M 1986. Dityrosine is a prominent component of the yeast ascospore wall: a proof of its structure. J Biol Chem 261: 4288-4294.

Christensen AM, Schaefer J, Kramer KJ, Morgan TD, Hopkins TL 1991. Detection of cross-links in insect cuticle by REDOR NMR spectroscopy. J Am Chem Soc 113: 6799-6802.

Davies MJ, Fu S, Wang H, Dean RT 1999. Stable markers of oxidant damage to proteins and their application in the study of human disease. Free Radic Biol Med 27: 1151-1163.

Dubey JP, Miller NL, Frenkel JK 1970. The Toxoplasma gondii oocyst from cat faeces. J Exp Med 132: 636-662.

Eschenbacher KH, Eggli P, Wallach M, Braun R 1996. Characterization of a $14 \mathrm{kDa}$ oocyt wall protein of Eimeria tenella and $E$. acervulina. Parasitology 112: 169-176.

Ferguson DJP, Belli SI, Smith NC, Wallach MG 2003. The development of the macrogamete and oocyst wall in Eimeria maxima: immunolight and electron microscopy. Int J Parasitol 33: 1329-1340.

Ferguson DJP, Brecht S, Soldati D 2000. The microneme protein MIC4, or an MIC4-like protein, is expressed within the macrogamete and associated with oocyst wall formation in Toxoplasma gondii. Int J Parasitol 30: 1203-1209.

Ferguson DJP, Hutchison WM, Siim JC 1975. The ultrastructural development of the macrogamete and formation of the oocyst wall of Toxoplasma gondii. Acta Pathol Microbiol Scand 83: 491-505.

Fetterer RH, Hill DE 1993. The occurrence of phenol oxidase activity in female Trichuris suis. J Parasitol 79: 155-159.

Fetterer RH, Rhoads ML 1990. Tyrosine-derived cross-linking amino acids in the sheath of Haemonchus contortus infective larvae. J Parasitol 76: 619-624.

Foerder CA, Shapiro BM 1977. Release of ovoperoxidase from sea urchin eggs hardens the fertilization membrane with tyrosine crosslinks. Proc Natl Acad Sci USA 74: 4214-4218. 
Fujimoto D 1975. Occurrence of dityrosine in cuticlin, a structure protein from Ascaris cuticle. Comp Biochem Physiol B Biochem Mol Biol 51: 205-207.

Huggins LG, Waite JH 1993. Eggshell formation in Bdelloura candida, an ectoparasitic turbellarian of the horseshoe crab Limulus polyphemus. J Exp Zool 265: 549-557.

Karim MJ, Basak SC, Trees AJ 1996. Characterization and immunoprotective properties of a monoclonal antibody against the major oocyst wall protein of Eimeria tenella. Infect Immun 64: $1227-1232$.

Kerwin LJ, Turecek F, Xu R, Kramer KJ, Hopkins TL, Gatlin CL, Yates JR 1999. Mass spectrometric analysis of catechol-histidine adducts from insect cuticle. Anal Biochem 268: 229-237.

Krücken J, Hosse RJ, Mouafo AN, Entzeroth R, Bierbaum S, Marinovski P, Hain K, Greif G, Wunderlich F 2008. Excystation of Eimeria tenella sporozoites impaired by antibody recognizing gametocyte/oocyst antigens GAM22 and GAM56. Eukaryot Cell 7: 202-211.

Kuticic V, Wikerhauser T 1996. Studies on the effect of various treatments on the viability of Toxoplasma gondii tissue cysts and oocysts. Curr Top Microbiol Immunol 219: 261-265.

LaBella F, Waykole P, Queen G 1968. Formation of insoluble gels and dityrosine by the action of peroxidase on soluble collagens. Biochem Biophys Res Commun 30: 333-338.

Lassandro F, Sebastiano M, Zei F, Bazzicalupo P 1994. The role of dityrosine formation in the crosslinking of CUT-2, the product of a second cuticlin gene of Caenorhabditis elegans. Mol Biochem Parasitol 65: 147-159.

McConville MJ, Thomas-Oates JE, Ferguson MA, Homans SW 1990. Structure of the lipophosphoglycan from Leishmania major. J Biol Chem 265: 19611-19623.

McLaren DJ 1969. Observations on the fine structural changes associated with schizogony and gametogony in Eimeria tenella. Parasitology 59: 563-574.

Meyers PR, Bourn WR, Steyn LM, Helden P, Beyers AD, Brown GD 1998. Novel method for rapid measurement of growth of mycobacteria in detergent-free media. J Clin Microbiol 36: 2752-2754.

Monné L, Hönig G 1954. On the properties of the shells of coccidian oocysts. Arkiv för Zoologi 7: 251-256.

Mouafo AN, Weck-Heimann A, Dubremetz J-F, Entzeroth R 2002. Monoclonal antibodies specific for the two types of wall-forming bodies of Eimeria tenella macrogametes (Coccidia, Apicomplexa). Parasitol Res 88: 217-224.

Nyberg PA, Bauer DH, Knapp SE 1968. Carbon dioxide as the initial stimulus for excystation of Eimeria tenella oocysts. J Protozool. 15: 144-148.

Nyberg PA, Knapp SE 1970. Effect of sodium hypochlorite on the oocyst wall of Eimeria tenella as shown by electron microscopy. Proc Helminthol Soc Wash 37: 32-36.

Page AP, Winter AD 2003. Enzymes involved in the biogenesis of the nematode cuticle. Adv Parasitol 53: 85-148.

Pittilo RM, Ball SJ 1979. The fine structure of the developing macrogamte of Eimeria maxima. Parasitology 79: 259-265.

Pittilo RM, Ball SJ 1980. The ultrastructural development of the oocyst wall of Eimeria maxima. Parasitology 81: 115-122.
Ryley JF 1973. Cytochemistry, physiology, and biochemistry. In DH Hammond, PL Long, The Coccidia: Eimeria, Isospora, Toxoplasma, and Related Genera, University Park Press, Baltimore, London, p. 145-181.

Ryley JF, Bentley M, Manner DJ, Stark JR 1969. Amylopectin, the storage polysaccharide of the coccidia Eimeria brunetti and $E$. tenella. J Parasitol 55: 839-845.

Schares G, Pantchev N, Barutzki D, Heydorn AO, Bauer C, Conraths FJ 2005. Oocysts of Neospora caninum, Hammondia heydorni, Toxoplasma gondii and Hammondia hammondi in faeces collected from dogs in Germany. Int J Parasitol 35: 1525-1537.

Scholtyseck E 1973. Ultrastructure. In DH Hammond, PL Long, The Coccidia: Eimeria, Isospora, Toxoplasma, and Related Genera, University Park Press, Baltimore, London, p. 81-144.

Scholtyseck E, Rommel A, Heller G 1969. Light and electron microscopic studies of the formation of the oocyst wall in Eimeria (Eimeria perforans, E. stiedae and E. tenella) Z Parasitenkd 31: 289-298.

Shirley MW 1995. Eimeria species and strains of chickens. In J Eckert, R Braun, MW Shirley, P Coudert, Biotechnology - Guidelines on techniques in Coccidiosis research, European Commission, Luxemburg, p. 1-51.

Smail EH, Briza P, Panagos A, Berenfeld L 1995. Candida albicans cell walls contain the fluorescent cross-linking amino acid dityrosine. Infect Immun 63: 4078-4083.

Spano F, Puri C, Ranucci L, Putignani L, Crisanti A 1997. Cloning of the entire COWP gene of Cryptosporidium parvum and untrastructural localization of the protein during sexual parasite development. Parasitology 114: 427-437.

Stotish RL, Wang CC, Meyenhofer M 1978. Structure and composition of the oocyst wall of Eimeria tenella. J Parasitol 64: 1074-1081.

Templeton TJ, Lancto CA, Vigdorovich V, Liu C, London NR, Hadsall KZ, Abrahamsen MS 2004. The Cryptosporidium oocyst wall protein is a member of a multigene family and has a homolog in Toxoplasma. Infect Immun 72: 980-987.

Thacker C, Peters K, Srayko M, Rose AM 1995. The bli-4 locus of Caenorhabditis elegans encodes structurally distinct kex $2 /$ subtilisin-like endoproteases essential for early development and adult morphology. Genes Dev 9: 956-971.

Thacker C, Rose AM 2000. A look at the Caenorhabditis elegans Kex2/Subtilisin-like proprotein convertase family. Bioessays 22: 545-553.

Thacker C, Sheps JA, Rose AM 2006. Caenorhabditis elegans dpy5 is a cuticle procollagen processed by a proprotein convertase. Cell Mol Life Sci 63: 1193-1204.

Thacker C, Srayko M, Rose AM 2000. Mutational analysis of bli-4/ $\mathrm{kpc}-4$ reveals critical residues required for proprotein convertase function in C. elegans. Gene 252: 15-25.

Waite JH 1990. The phylogeny and chemical diversity of quininetanned glue and varnishes. Comp Biochem Physiol B Biochem Mol Biol 97: 19-29.

Wallach M 2002. The development of CoxAbic ${ }^{\circledR}$ a novel vaccine against coccidiosis. World Poultry 18: 24-26.

Xu R, Huang X, Hopkins TL, Kramer KJ 1997. Catecholamine and histidyl protein cross-linked structures in sclerotized insect cuticle. Insect Biochem Mol Biol 27: 101-108. 